\title{
Economic Impact of Microarray-Based Rapid Detection of Gram-Positive Organisms in Blood Cultures
}

\author{
Paul Mariani, Jesse Breidenbach and Felix Roth* \\ Sanford Health Fargo, SU Laboratory, USA
}

\begin{abstract}
We assessed the clinical and economic impact of using the microarray-based Verigene Gram positive blood culture (BC-GP) test to identify gram positive bacteria in blood cultures. The main advantage of employing this test in comparison to conventional culture techniques resided on identifying the causative organism an average of 42.8 hours prior than conventional culture techniques. The microarray-based test results allowed providers to tailor antibiotic therapy and decide whether to admit/discharge a patient sooner, which reflected over $\$ 21,000$ in hospital savings for the 105 cases analyzed in this study. The implementation of the Verigene BC-GP in our Microbiology Laboratory presented an economic advantage when compared to conventional workup and was shown to improve ongoing antimicrobial stewardship and patient care.
\end{abstract}

Keywords: Sepsis; Gram positive; Organism; Microarray; Antibiotic; Stewardship

\section{Introduction}

Bloodstream infections (BSI) constitute a growing public health concern in the industrialized world. With an associated death toll of approximately 36,000 individuals per year, BSIs ranked as the $11^{\text {th }}$ leading cause of death in the US [1]. In addition to being an important cause of morbidity and mortality, BSIs are an economic burden for healthcare institutions and patients [2-4].

By identifying the causative organism and performing antibiotic susceptibilities testing (AST), the laboratory provides crucial information to guide the correct treatment for patients with BSIs [5]. In spite of being the gold standard, culture-based methods for identification and antibiotic susceptibilities are time consuming, which leads to patients not receiving specific antibiotic therapy promptly, increasing patient and hospitals' costs by means of inappropriate therapy and longer stay [2]. In this era of drug resistant pathogens, practitioners are often obligated to prescribe broad spectrum antibiotics, which are often more expensive, may be associated with drug toxicities necessitating monitoring, and in worst case scenarios lead to additional antimicrobial resistance [6,7]. Furthermore, incorrect antibiotic therapy administration has been shown to be associated with increased risk of mortality, particularly during the first 24 hours of infection [8]. With the advent of molecular techniques developed to detect bacterial genetic information, the turnaround time of pathogen identification and markers of resistance has been greatly reduced $[9,10]$. Nevertheless, many of these molecular techniques require a certain level of expertise to be executed in the routine microbiology laboratory $[11,12]$. In recent years, microarray-based tests have emerged as a strong and reliable alternative to PCR-based methods [13]. In our study we focused on implementing a user-friendly microarray-based platform. Previous studies have evaluated the performance of such platform $[14,15]$, but only one paper to date has focused on the economic impact of Enterococci [16] detection. In addition, studies focused on the economic value of detection of multiple gram-positive organisms using this microarray technology are lacking. We sought to assess the impact of using the Verigene BC-GP test results on the following parameters: antibiotic therapy intervention, drug monitoring decisionmaking, decision to admit or discharged based on pathogenicity or the organism.

\section{Materials and Methods}

\section{Instrumentation}

We utilized the Verigene Gram-positive blood culture (BC-GP) test, a microarray-based platform with random access (each sample can be run independently without the need of batching) which identifies three Gram-positive genus targets (Staphylococcus spp., Streptococcus spp., Listeria spp.), nine Gram-positive species-level targets (S. aureus, S. epidermidis, S. lugdunensis, S. pneumoniae, S. agalactiae, S. pyogenes, S. anginosus Group, E. faecalis, E. faecium), and detects the genetic resistance markers mecA and $\operatorname{van} A / \operatorname{van} B$. This test is FDA approved for clinical use. Concomitantly, conventional blood culture techniques (considered as the reference method) were performed.

\section{Clinical Validation}

In order to initiate our study, we performed a clinical validation in which 30 samples from positive blood cultures containing at least one Gram-positive organism/resistance marker were tested by the Verigene BC-GP. Prior to commencing our study we performed a validation by testing at least two positive samples for each organism/ resistance marker detected by the BC-GP test; we obtained a $100 \%$ concordance. Due to the low incidence of BSI caused by Listeria spp. In our institution we did not include this organism in our validation/ study despite Verigene BC-GP's ability to detect this organism.

\section{Samples Testing}

Between February and September 2013, blood cultures submitted to our microbiology laboratory and positive by the BacT/ALERT system (BioMérieux, Durham, NC), which were subsequently gram stained

*Corresponding author: Felix Roth, Sanford Health Fargo, SU Laboratory. 1720 S University Dr, Fargo, ND 58103, USA, Tel: 17012804873; E-mail: felix. roth@sanfordhealth.org

Received May 05, 2015; Accepted June 22, 2015; Published June 24, 2015

Citation: Mariani P, Breidenbach J, Roth F (2015) Economic Impact of MicroarrayBased Rapid Detection of Gram-Positive Organisms in Blood Cultures. J Med Microb Diagn S3: 009. doi:10.4172/2161-0703.S3-009

Copyright: (c) 2015 Mariani $P$, et al. This is an open-access article distributed under the terms of the Creative Commons Attribution License, which permits unrestricted use, distribution, and reproduction in any medium, provided the original author and source are credited. 
and determined to be gram-positive cocci (GPC), were included in the study. Samples originated from inpatients and outpatients seen at our community hospital serving the population of Fargo, ND.

Only the first positive bottle for each patient with de novo bacteremia was included. Patients with history of bacteremia within the preceding six months were not included in the study this decision was driven by our laboratory policy which reports MRSA infections as new for patients who were not positive for this organism in the prior six months. For the samples that showed positivity but not true bacteremia (presence of viable bacteria in the bloodstream) was found, the information was also accounted since contamination of blood cultures (mainly with coagulase negative staphylococcus [CoNS]), along with other clinical aspects, can drive the decision of admitting or discharging a patient.

Following Gram stain, both conventional workup and the BCGP test were set up. In the case of conventional workup, culture was performed on blood agar plates (BAP) and Columbia Nalidixic Acid (CNA) to obtain isolated colonies; after growth, S. aureus, CoNS and beta-hemolytic Streptococcus species were identified by spot testing using Staphaurex or PathoDx kits respectively (Thermo-Fisher Scientific Remel Products, Lenexa, KS) and/or Vitek-2 ID cards (BioMérieux, Durham, NC), all other GPC were identified by Vitek-2 ID cards. Antibiotic susceptibility testing (AST) was carried out using Vitek-2 AST cards or the Etest diffusion method (BioMérieux, Durham, NC). Since the BC-GP test only provides information regarding presence of genetic markers for resistance (which does not replace full antibiotic susceptibilities) the AST values were used to compare cases in which resistance markers were found, for the rest of the results only time to generate a species level identification was taken into account.

In order to calculate the turnaround time difference between BC-GP and conventional workup, we considered time zero to be the moment when the blood culture bottles were introduced in the BacT/ ALERT system (which on average is up to a maximum of 30 minutes after collection per our microbiology laboratory receiving station). We believe this to be a more realistic approach that better reflects a true microbiology laboratory routine and eliminates taking into account any delays that may have occurred when unloading the positive bottles or performing the Gram stain.

\section{Intervention and analysis based on BC-GP results}

The standard process for positive blood cultures reporting in our laboratory consists on performing the Gram stain and report the result (considered as a critical value) to the primary care team/RN as soon as available; subsequently the identification results and AST would be electronically reported as obtained, and retrieved by the physician when available.

In our study the physician/RN received a call with the Gram stain result as usual; after approximately three hours (the time it takes the BC-GP test to run), an infectious diseases doctor was called with the BC-GP test results, he promptly communicated the findings to the physician seeing the patient associated with the sample in question. The rationale of having the infectious disease doctor communicating the $\mathrm{BC}-\mathrm{GP}$ result was delivering the results in the most effective manner possible. This practice was changed to reporting the BC-GP results directly to the $\mathrm{RN} /$ primary care team after completion of the study.

After completion of the Verigene BC-GP test for this study (September 2013), an infectious disease doctor retrospectively accessed patients' charts and investigated if decisions on treatment, admission, or discharge were made based upon the BC-GP results (to determine if actions were taken based on BC-GP, the chart notes had to clearly state the use of the BC-GP test).

Using this data set, a pharmacist calculated the costs for treatment changes and drug monitoring using the correct dosing calculation for each patient; being the main parameters considered weight, renal function and site of infection. These are parameters analyzed constantly by our antibiotic stewardship program run by the pharmacy department along with the infectious diseases department to make suggestions on therapy.

The design, procedures, and materials and methods were approved by our Institutional Review Board (IRB).

For statistical analysis, we employed a Mann-Whitney U-test (Sigma Plot v 11.2.0, Systat Software Inc.), considering a p value of 0.05 as significant.

\section{Results}

A total of 107 blood culture samples containing gram positive cocci were tested by conventional workup and the BC-GP test. Both methods identified one organism per sample (107 organisms) from which 105 (98.1\%) showed correlation; the two organisms that were misidentified by BC-GP were Streptococcus mitis (identified as S. pneumoniae by BC-GP) and Aerococcus viridians (identified as Staphylococcus spp. by BC-GP). Therefore, the overall sensitivity of BC-GP to detect GPC was $100 \%\left(\mathrm{IC}_{95}=98.3 \pm 1.7 \%\right)$, whereas specificity was $98.2 \%\left(\mathrm{IC}_{95}=\right.$ $96.6 \pm 3.1 \%)$. With respect to specific organisms, coagulase-negative Staphylococci (CoNS) were the most frequently found GPC $(39.3 \%)$, followed by S. aureus (18.1\%), S. pneumoniae (8.4\%) and Enterococci (5.6\%). The mecA resistance gene for $S$. aureus was detected by both BC-GP and the reference method in 16 of 20 total $S$. aureus isolates recovered, showing specificity and sensitivity of $100 \%$ by the BCGP test. Similar results were found in the most frequently detected organisms.

Regarding time to organism identification and result reporting, when comparing the reference method to BC-GP, there was a consistent earlier identification time for BC-GPThe average time to identification for each individual organism was reduced on average by 43.0 hours using BC-GP (Table 1). This difference was shown to be statistically significant $(\mathrm{p}<0.01)$.

The last portion of the investigation consisted of analyzing the

\begin{tabular}{|c|c|c|c|c|}
\hline & \multicolumn{3}{|c|}{ Average time to identification } & \\
(in hours) & \\
\hline Organism & Verigene & Conventional & Difference & p value \\
\hline MRSA & 38.5 & 106.1 & 67.6 & $<0.001$ \\
\hline MSSA & 23.3 & 64.0 & 40.7 & $<0.001$ \\
\hline CoNS & 39.0 & 82.7 & 43.8 & $<0.001$ \\
\hline Streptococcus spp* & 33.7 & 63.4 & 29.8 & $<0.005$ \\
\hline GAS & 22.2 & 42.9 & 20.7 & $\begin{array}{c}\text { N/A (small } \\
\text { sample) }\end{array}$ \\
\hline GBS & 21.4 & 46.0 & 24.6 & $<0.01$ \\
\hline S. pneumoniae & 20.3 & 41.9 & 21.6 & $<0.001$ \\
\hline Enterococcus spp & 52.3 & 104.4 & 52.1 & $<0.05$ \\
\hline $\begin{array}{c}\text { All Organisms } \\
\text { (n=105) }\end{array}$ & 34.5 & 77.5 & 43.0 & $<0.01$ \\
\hline
\end{tabular}

*S. anginosus group considered as Streptococcus spp

Table 1: Time to detection per organism comparison between BC-GP and conventional methods. 
patient's charts and determining the proportion of patients for which the results of BC-GP impacted antibiotic (ATB) therapy, drug monitoring and discharge/admission decisions; the associated cost to each event was also calculated (Table 2). The three antibiotics used to treat patients in our study for which savings were calculated were vancomycin, daptomycin and linezolid. The drug monitoring test was performed for patients receiving vancomycin.

There were a total of 37 (35.4\%) cases in which ATB therapy was changed. There were 5 cases $(4.8 \%)$ in which a discharge/admission decision was rendered based on the BC-GP test results. Vancomycin was avoided from being prescribed as initial therapy in $22(21 \%)$ of the patients whereas this antibiotic was discontinued upon notification of BC-GP results for 11 (10.5\%) patients. Vancomycin avoidance/ discontinuation sub-groups were found to be positive for CoNS ( 9 , $8.6 \%)$, MSSA $(8,7.6 \%)$ or Streptococcus spp. $(4,3.8 \%)$. The total savings for discontinuing vancomycin therapy in favor of a cephalosporin, including associated drug monitoring, was $\$ 1,545.02$. There were three $(2.9 \%)$ patients, who had prior history of VRE colonization; in our institution daptomycin is the empiric antibiotic of choice for these patients if blood cultures are found to contain GPC, nevertheless, BCGP allowed to promptly detect CoNS or S. anginosus, which allowed the physicians to avoid daptomycin treatment and use vancomycin or a cephalosporin instead, representing savings of $\$ 2,565.36$. Similar to patients with history of VRE, patients known to be colonized with MRSA and finding of GPC in the blood are empirically treated at our institution with an antimicrobial agent against MRSA. For one case (1\%) S pneumonia BSI was found in a patient colonized with MRSA. Per notes, the physician had planned on starting linezolid (patient was on levofloxacin and metronidazole); yet when results became available a cephalosporin was used in its place. This was associated with a cost savings of $\$ 114.26$ (Table 2). Three cases of outpatients with GPC in blood were planned on being admitted by emergency room and oncology service. With the finding of CoNS by BC-GP, these services opted not to admit the patient. The cost savings was calculated based upon the time the patient would have been hospitalized while conventional method finalized result as CoNS. Similarly, one patient was discharged earlier from the hospital because a blood isolate was confirmed as CoNS by BC-GP. The total savings for this sub-group of four patients $(3.8 \%$ ) was $\$ 17,152.00$ based on the current rates (year 2013) per inpatient stay in our hospital. Decision on admission to the hospital or early discharge was calculated without factoring in the potential cost savings from length-of-stay reduction, the overall savings for the 105 cases studied was $\$ 21,376.64$ (Table 2).

\section{Discussion}

The study presented aimed to depict a realistic situation of implementing a rapid molecular test for the detection of BSI caused by GPC. Previous studies, a majority of which have been based on employing qPCR [17-19], but many of these studies were mainly focused on a smaller set of organisms [20,21]. From the technical

\begin{tabular}{|c|c|c|c|}
\hline & $\mathbf{N}^{\circ}$ of patients & Percentage & Cost Savings \\
\hline Vancomycin avoided & 22 & $21.0 \%$ & $\$ 717.06$ \\
\hline Vancomycin discontinued & 11 & $10.5 \%$ & $\$ 269.06$ \\
\hline Drug monitoring avoided & N/A & N/A & $\$ 558.90$ \\
\hline Daptomycin not added & 3 & $2.9 \%$ & $\$ 2,565.36$ \\
\hline Linezolid not added & 1 & $1.0 \%$ & $\$ 114.26$ \\
\hline No admission/Early discharge & 5 & $4.8 \%$ & $\$ 17,152.00$ \\
\hline Total & $\mathbf{4 2}$ & $\mathbf{4 0 . 2} \%$ & $\mathbf{\$ 2 1 , 3 7 6 . 6 4}$ \\
\hline
\end{tabular}

Table 2: Cost savings associated with BC-GP results. point of view, we also believe that the system evaluated in this study also presents an advantage over PCR-based methods; since it detects pre-existing DNA from the organisms present in the sample and lacks amplification steps, it is not be subject to carryover contamination [22]. Newer technologies such as MALDI-TOF [23] have also been shown to improve time to identification over conventional culturebased techniques when testing directly from positive blood cultures; nevertheless, they require additional steps to prepare the sample for analysis, which ultimately leads to a time to result similar to the BC-GP test [24]. Another limitation of MALDI-TOF lies in that it usually has to be combined with additional testing methods in order to obtain a resistance profiles, such as for the identification of MRSA [25].

A considerable number of the publications available addressing economic impact associated with BSIs are based on estimates, not direct analysis, of outcomes based on test results [26,27]. Despite providing invaluable information, we felt that a more direct approach to capturing economic outcomes was needed.

Even though full susceptibility profiles cannot be provided by the Verigene BC-GP test, the ability to detect genetic markers to identify MRSA or VRE is nonetheless a very useful feature. The ability to rule out the presence of organisms such as VRE in previously known colonized patients also proved to be useful in avoiding administration of expensive antibiotics such as daptomycin.

Despite not performing a length of stay (LOS) analysis in the present work, we could infer that there may be potential additional benefits in this aspect based on previous published data showing the advantages of rapid identification methods over LOS [28-31]. Nevertheless, we would have to perform specific studies to reflect the specific LOS outcome in our institution based on the use of the BC-GP test.

We have demonstrated that a multiplex test based on microarray technology can be very sensitive and specific, improve turnaround time to report results, which in turn was translated into significant hospital savings. Finally, the ability of directly affecting treatment by allowing providers to administer appropriate ATB sooner makes the Verigene BC-GP microarray-based system a desirable addition to the laboratory in order to contribute to achieve the ultimate goal of the hospital, improving patient care.

\section{Acknowledgements}

The authors would like to thank to the Microbiology Laboratory staff at Sanford Health Fargo for their collaboration in the execution of this study.

\section{References}

1. Hoyert DL, Jiaquan X (2012) Deaths: Preliminary Data from 2011. Natl Vital Stat Rep 61: 1-51.

2. Kaye KS, Marchaim D, Chen TY, Baures T, Anderson DJ, et al. (2014) Effect of nosocomial bloodstream infections on mortality, length of stay, and hospital costs in older adults. J Am Geriatr Soc 62: 306-11.

3. Niven DJ, Fick GH, Kirkpatrick AW, Grant V, Laupland KB (2010) Cost and outcomes of nosocomial bloodstream infections complicating major traumatic injury. J Hosp Infect 76: 296-9.

4. Scheetz MH, Bolon MK, Postelnick M, Noskin GA, Lee TA (2009) Costeffectiveness analysis of an antimicrobial stewardship team on bloodstream infections: a probabilistic analysis. J Antimicrob Chemother 63: 816-25.

5. Coghe $F$, Orrù $G$, Pautasso $M$, Ferraguti $P$, Sanna $P$, et al (2011) The role of the laboratory in choosing antibiotics. J Matern F et al. Neonatal Med Suppl 2: 18-20.

6. Kane-Gill SL, Jacobi J, Rothschild JM (2010) Adverse drug events in intensive care units: risk factors, impact, and the role of teamcare. Crit Care Med 38: S83-9. 
Citation: Mariani P, Breidenbach J, Roth F (2015) Economic Impact of Microarray-Based Rapid Detection of Gram-Positive Organisms in Blood Cultures. J Med Microb Diagn S3: 009. doi:10.4172/2161-0703.S3-009

7. Spicknall IH, Foxman B, Marrs CF, Eisenberg JN (2013) A modeling framework for the evolution and spread of antibiotic resistance: literature review and model categorization. Am J Epidemiol 178: 508-20.

8. Kumar A, Ellis P, Arabi Y, Roberts D, Light B, et al. (2009) Initiation of inappropriate antimicrobial therapy results in a fivefold reduction of survival in human septic shock. Chest 136: 1237-48.

9. Bauer KA, West JE, Balada-Llasat JM, Pancholi P, Stevenson KB, et al. (2010) An antimicrobial stewardship program's impact with rapid polymerase chain reaction methicillin-resistant Staphylococcus aureus/S. aureus blood culture test in patients with S. aureus bacteremia. Clin Infect Dis 51: 1074-80.

10. Wang HY, Kim S, Kim H, Kim J, Kim Y, et al. (2014) Real-time PCR TaqMan assay for rapid screening of bloodstream infection. Ann Clin Microbio Antimicrob 13: 3.

11. Hansen WL, Beuving J, Verbon A, Wolffs PF (2012) One-day workflow scheme for bacterial pathogen detection and antimicrobial resistance testing from blood cultures. J Vis Exp 65.

12. Karumaa S, Kärpänoja P, Sarkkinen H (2012) PCR identification of bacteria in blood culture does not fit the daily workflow of a routine microbiology laboratory. $\mathrm{J}$ Clin Microbiol 50: 1031-3

13. Liesenfeld O, Lehman L, Hunfeld KP, Kost G (2014) Molecular diagnosis of sepsis: New aspects and recent developments. Eur J Microbiol Immunol 4:1-25.

14. Mestas J, Polanco CM, Felsenstein S, Dien Bard J (2014) Performance of the Verigene Gram-positive blood culture assay for direct detection of Grampositive organisms and resistance markers in a pediatric hospital. J Clin Microbiol 52: 283-7.

15. Buchan BW, Ginocchio CC, Manii R, Cavagnolo R, Pancholi P, et al. (2013) Multiplex identification of gram-positive bacteria and resistance determinants directly from positive blood culture broths: evaluation of an automated microarray-based nucleic acid test. PLoS Med 10: e1001478.

16. Sango A, McCarter YS, Johnson D, Ferreira J, Guzman N, et al. (2013) Stewardship approach for optimizing antimicrobial therapy through use of a rapid microarray assay on blood cultures positive for Enterococcus species. J Clin Microbiol 51: 4008-11.

17. Paolucci M, Stanzani M, Melchionda F, Tolomelli G, Castellani G, et al. (2013) Routine use of a real-time polymerase chain reaction method for detection of bloodstream infections in neutropaenic patients. Diagn Microbiol Infect Dis 75 $130-4$

18. Ozbak H, Dark P, Maddi S, Chadwick P, Warhurst G (2012) Combined molecula gram typing and high-resolution melting analysis for rapid identification of a syndromic panel of bacteria responsible for sepsis-associated bloodstream infection. J Mol Diagn 14: 176-84.
19. Haag H, Locher F, Nolte O (2013) Molecular diagnosis of microbial aetiologies using SepsiTest ${ }^{\mathrm{TM}}$ in the daily routine of a diagnostic laboratory. Diagn Microbio Infect Dis 76: 413-8.

20. Palavecino EL (2014) Rapid methods for detection of MRSA in clinical specimens. Methods Mol Biol. 1085: 71-83.

21. Wang HY, Kim S, Kim J, Park SD, Uh Y, et al. (2014) Multiplex Real-time PCR Assay for the Rapid Detection of Methicillin-Resistant Staphylococci Directly from Positive Blood Cultures. J Clin Microbiol 52: 1911-20.

22. Aslanzadeh J (2004) Preventing PCR Amplification Carryover Contamination in a Clinical Laboratory. Ann Clin Lab Sci 34: 389-96.

23. Foster AG (2013) Rapid Identification of microbes in positive blood cultures by use of the vitek MS matrix-assisted laser desorption ionization-time of flight mass spectrometry system. J Clin Microbiol. 51: 3717-9.

24. Wang MC, Lin WH, Yan JJ, Fang HY, Kuo TH, et al. (2013) Early identification of microorganisms in blood culture prior to the detection of apositive signal in the BACTEC FX system using matrix-assisted laserdesorption/ionization-time of flight mass spectrometry. J Microbiol Immunol Infect pii: S1684-1182(13)00200-4.

25. Clerc O, Prod'hom G, Senn L, Jaton K, Zanetti G, et al. (2014) Matrix-assisted laser desorption ionization time-of-flight mass spectrometry and PCR-based rapid diagnosis of Staphylococcus aureus bacteraemia. Clin Microbiol Infect 20: 355-60.

26. Leibovici L, Paul M, Andreassen S (2010) Balancing the benefits and costs of antibiotic drugs: the TREAT model. Clin Microbiol Infect 16: 1736-9.

27. Theodoro D, Owens PL, Olsen MA, Fraser V (2014) Rates and timing of central venous cannulation among patients with sepsis and respiratory arrest admitted by the emergency department. Crit. Care Med 42: 554-64.

28. Zwang O, Albert RK (2006) Analysis of strategies to improve cost effectiveness of blood cultures. J Hosp Med 5: 272-6.

29. Galar A, Yuste JR, Espinosa M, Guillén-Grima F, Hernáez-Crespo S, et al. (2012) Clinical and economic impact of rapid reporting of bacterial identification and antimicrobial susceptibility results of the most frequently processed specimen types. Eur J Clin Microbiol Infect Dis 9: 2445-52.

30. Wong JR, Bauer KA, Mangino JE, Goff DA (2012) Antimicrobial stewardship pharmacist interventions for coagulase-negative staphylococci positive blood cultures using rapid polymerase chain reaction. Ann Pharmacother 46: 1484-90.

31. Huang AM, Newton D, Kunapuli A, Gandhi TN, Washer LL, et al. (2013) Impact of rapid organism identification via matrix-assisted laser desorption/ionization time-of-flight combined with antimicrobial stewardship team intervention in adult patients with bacteremia and candidemia. Clin Infect Dis. 57: 1237-45. 